\title{
System of recognition of an accessory of persons to racial and an ethnic group on the basis of photographs
}

\author{
Tofiq Kazimov, Shafagat Mahmudova ${ }^{1, *}$, Tugrul Aktas \\ Accepted $15^{\text {th }}$ August 2014 \\ DOI: 10.18100/ijamec.71990
}

\begin{abstract}
This article is devoted to developing a recognition method of race and ethnicity of individual based on portrait photographs. The reference image is formed based on selected geometric points of the face and a special algorithm for calculating the characteristic parameters of the images available in the database. Next, the original image is compared with the reference images of ethnic groups, and thus, the affiliation of the original image to a specific ethnic group is determined.
\end{abstract}

Keywords: Recognition, race, ethnicity, ethnic group, racial origin, identification points.

\section{Introduction}

Identification problem based on race and ethnicity is one of the topical problems in the developing information society. Currently, specialists of different scientific fields are engaged in this issue. Existing problems in this field are solved in different forms. Different methods are used in solution of these problems. Ethnography is engaged in solution of the problems.

Races were formed during the evolution of the humanity and are distinguished in certain geographical territories where they are spread. Issues related to races and their primary division is solved in different forms and selection of indications depends on the used system.

Race is a system of human groups that combine genetic biological signs and external similarities formed in a certain geographical region. Characteristic lines of different races are possible as a result of adaptation of people to a certain environment.

Generally, human faces are divided in three racial clusters [1]:

1. mongoloid;

2. avropoid;

3. negroid.

Research of classic anthropology proves the existence of 2 poles in this field:

1. east;

2. west.

Currently scientists distinguish 30 human races.

As division of three races - white, yellow and black is an outdated position, currently scientists distinguish 30 human races combines in three groups called great races.

Ethnicity is a group of people with relatively constant characteristics, culture and psychology, as well as distinguishing their unity from other human groups and speaking the same language. Ethnical identification is a comparison process of the subjects belonging to a certain ethnic group with the subjects of

\footnotetext{
${ }^{1}$ Institute of Information Technology of ANAS, Azerbaijan.

*Corresponding Author: Email: shafagat_57@mail.ru

\# This paper has been presented at the International Conference on

Advanced Technology\&Sciences (ICAT'14) held in Antalya (Turkey),

August 12-15, 2014
}

another ethnic group. Recognition of a human face according to race and ethnicity based on the images is a topical task that requires conducting new researches.

A new method is proposed to create a real three-dimensional portrait photograph based on the two-dimensional portrait for synthesis of two dimensional portrait photographs arbitrarily made in some works using special models prepared in accordance with gender and ethnicity [3].

Ethnicity is considered as a very important demographic characteristic for the humanity. Automatic ethnic classification based on huma $\mathrm{n}$ face is important in different fields. Some articles have research both two- and three-dimensional characteristics of the human face, MM-LBP multi-modal method (multi- scale multi-ratio) has been proposed for ethnic classification. LBP (Local Binary Pattern) histograms were compiled from multi-scale multi-ratio quadrangular parts based on texture and range images [4].

Visual information such as gender, age, ethnicity and facial expression play an important role in face-to-face communication. Some articles have proposed a new approach for ethnic classification of the human face based on the portrait photograph. Gabor Wavelets Transformation identifying the main facial expressions and eye reticular membrane samples were combined in this approach. Support vector apparatus was used for ethnic classification. Using the system based on this approach, 94\% ethnic evaluation was achieved in different lighting conditions [5].

Unlike existing models depending only on two-dimensional portrait photographs in some articles, a new method was proposed for solution of issues related to combination of local texture and form characteristics obtained using three-dimensional models of the face. Proposed method used Oriented Gradient Maps in order to demonstrate local geometric variations, as well as all texture variations of the face [6].

The article is devoted to developing a method to determine the generalized geometric facial characteristics based on portraits of people who have the same historical and ethnic features. Base is formed on the basis of portrait photographs of people affiliated with the same racial, tribal, ethnic group in three generations. In the future, for simplicity, they will be called ethnic groups of 
people. The reference image is formed based on selected geometric points of the face and a special algorithm for calculating the characteristic parameters of the images available in the database, i.e. the basic matrix of geometric characteristics of the reference image. Identification task is reduced to comparing the original image with the reference image. It must be noted that during the identification, such important and significant physical features such as skin color, hair color and texture, color and shape of the eye are not taken into account. However, upon developing the identification system, these factors can be considered as additional stationary parameters that improve recognition effectiveness.

\section{Problem statement}

The human face is the source of various information signals that are interpreted by others for recognition. Face can provide information about age, gender, ethnic or racial origin of its owner. Identification of the human person is carried out almost instantly. Upon recognizing a person, a facial image (portrait photograph) is of particular theoretical and practical value $[1,2,3]$. There is currently a significant number of articles devoted to recognize people based on portrait photographs $[8,9,10,11,12]$. However, there are much less works on the recognition of people based on their affiliation to an ethnic group is much less $[1,2,3,4,5,6,7]$.

Apparently, people affiliated with different ethnical groups are significantly different from each other by from and placements of facial patterns (facial parts).

In order to start the identification task, primarily we must determine the parameters of the original image that must be operated on.

It is necessary to define the set of characteristic (identification) points of the human face and based on them - many signs (characteristic parameters) based on which the identification will be conducted. At the same time, of course, some requirements for portraits must be taken into account:

- identification points must not be obstructed with hair, beard, mask, etc.;

- recognition process should not depend on the scale of the portrait;

- a system of identification points will ensure a relative stability of the recognition process upon a little change of camera angle (light head rotation, tilt, facial expressions, etc.);

- the number of identification points shall be the maximum (if possible) to ensure high recognition accuracy.

This article stipulates the development of a special algorithm for the formation of the reference image and identification of the original image for its affiliation with in a particular ethnic group.

\section{Solution algorithm}

On the basis of conducted studies on the analysis of existing development in the literature available in this field $[1,2,3,7]$ and own experience $[8,9,10]$, it was concluded that it is necessary to provide about 30 identification points on the human image. These points must be invariant to small changes (angle, lighting, facial expressions, cosmetics, age-related) of images.

During conduction of preliminary experiments, it was decided to start with 30 following identification points, on the assumption that a face is considered symmetrical in accordance with a line that divides the face into two symmetrical halves as shown in figure 1:

- $a_{1}-$ tip of the chin;
- $a_{2}$ - bottom point of the lower lip;

- $a_{3}$ - center of the mouth, as the occlusion point of lips on the facial symmetry line;

- $a_{4}$-right corner of the mouth;

- $a_{5}$ - far right point of the face on mouth line level;

- $a_{6}$ - far point of the upper lip on face symmetry line;

- $a_{7}-$ far lower point of the nose;

- $a_{8}$ - tip of the nose (on the nose line);

- $a_{9}$ - far right point of the nose on the nose line;

- $a_{10}$ - far right point of the face on the nose line;

- $a_{11}$ - middle point of the portion adjoining the centers of eye pupils;

- $a_{12}-$ left corner of the right eye;

- $a_{13}$-point of the lower lid of the right eye laying perpendicular to the eye line passing through the pupil of the right eye;

- $a_{14}$ - right corner of the right eye;

- $a_{15}$ - far right point of the face on the eye line;

- $a_{16}$-point of the upper lid of the right eye laying perpendicular to the eye line passing through the pupil of the right eye;

- $a_{17}-$ point of the right lid of the right eye laying perpendicular to the eye line passing through the pupil of the right eye;

- $a_{18}$ - far right point of the face on the brow line;

- $a_{19}$ - far upper point of the forehead on the facial symmetry line;

- $a_{20}$ - point determining the center of the pupil of the right eye.

- $a^{*}{ }_{4}-$ left corner of the mouth;

- $a^{*}{ }_{5}-$ far left point of the face on the mouth line level;

- $a^{*}{ }_{9}$ - far left point of the nose on the nose line;

- $a^{*}{ }_{10}-$ far left point of the face on the nose line;

- $a_{12}^{*}$ - right corner of the left eye;

- $a^{*}{ }_{14}-$ left corner of the left eye;

- $a^{*}{ }_{15}$ - far left point of the face on the eye line;

- $a^{*}{ }_{17}-$ point of the left lid of the left eye laying perpendicular to the line;

- $a^{*}{ }_{18}$ - far left point of the face on the brow line;

- $a_{20}^{*}$ - point determining the center of the pupil of the eye. where

$\checkmark$ facial symmetry line - is a line dividing the face in two symmetrical halves;

$\checkmark$ mouth line - perpendicular to the symmetry line, passing through the center of the mouth;

$\checkmark$ nose line - perpendicular to the symmetry line passing through the tip of the nose;

$\checkmark$ eye line - line passing through the centers of the eye;

$\checkmark \quad$ brow line - line passing through the points $a_{17}$ and $a^{*}{ }_{17}$.

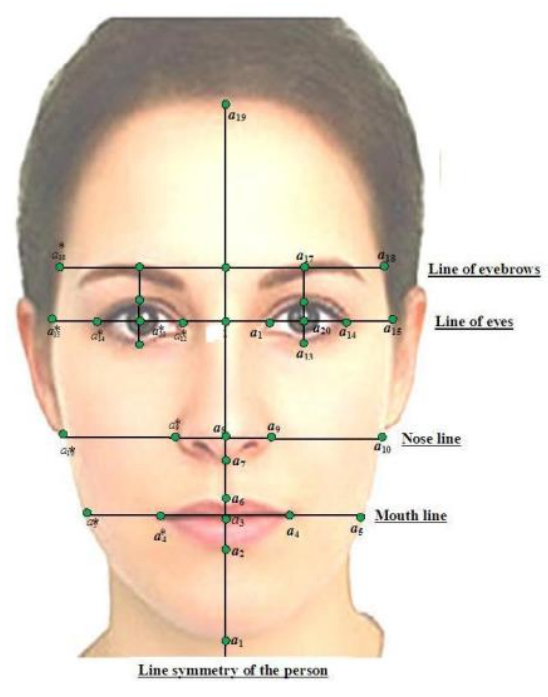


Figure.1. Identification points on the face of human Let's form a distance matrix $S\left(s_{i j} ; i=1,20 ; j=1,20\right)$ for points $a_{1}$ ,..., $\quad a_{20}$ as following (fig.2): $s_{i j}=\operatorname{dist}\left(a_{i}, a_{j}\right)$, for $i<j, s_{i j}=0$, for $i \geq j$, where $\operatorname{dist}\left(a_{i}, a_{j}\right)-$ is the Euclidean distance between the points of $a_{i}$ and $a_{j}$. Additionally, let's determine following distances:

$s_{1}{ }^{*}=\operatorname{dist}\left(a_{4}, a^{*}{ }_{4}\right), s_{2}{ }^{*}=\operatorname{dist}\left(a_{5}, a^{*}\right)$,

$s_{3}{ }^{*}=\operatorname{dist}\left(a_{9}, a_{9}{ }_{9}\right), s_{4}{ }^{*}=\operatorname{dist}\left(a_{10}, a^{*}{ }_{10}\right)$,

$s_{5}{ }^{*}=\operatorname{dist}\left(a_{12}, a^{*}{ }_{12}\right), s_{6}{ }^{*}=\operatorname{dist}\left(a_{14}, a^{*}{ }_{14}\right)$,

$s_{7}{ }^{*}=\operatorname{dist}\left(a_{15}, a^{*}{ }_{15}\right), s_{8}{ }^{*}=\operatorname{dist}\left(a_{18}, a^{*}{ }_{18}\right), s_{9}{ }^{*}=\operatorname{dist}\left(a_{20}, a^{*}{ }_{20}\right)$.

Let's compose the vector $V\left(v_{k}, k(i, j)=1,190\right)$ based on non-zero entries of the $S$ matrix as following:

$v_{k}=s_{i j}, \quad i=1,19 ; j=i+1,20$,

where

$k(i, j)=20(i-1)+j-i(i+1) / 2$

If all coordinates of the $V\left(v_{k}, k(i, j)=1,190\right)$ vector are divided subsequently to $s_{i}{ }^{*}(i=1,9)$, we will acquire the matrix of characteristics parameters of the image $W\left(w_{i k}\right.$ $i=1,9 ; k(i, j)=1,190)$, where

$\mathrm{w}_{\mathrm{ik}}=\mathrm{v}_{\mathrm{k}} / \mathrm{s}_{\mathrm{i}}^{*}, \mathrm{i}=1,9 ; \mathrm{k}(\mathrm{i}, \mathrm{j})=1,190$.

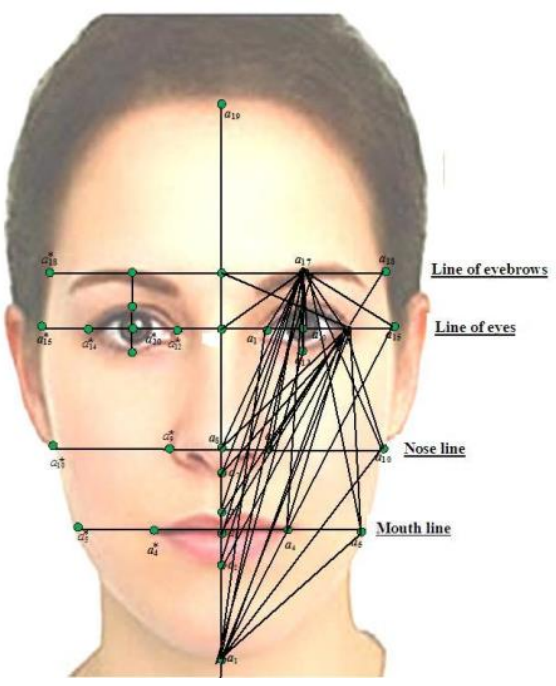

Figure 2. Distance for points on the face of human

As is known, the characteristic parameters as ratios for identification parameters of images make them scale-independent of the distance from which a photograph of the person is taken. Besides, the actual dimensions of the facial patterns and the distance between them do not matter for recognition $[11,12,13]$.

Now, let's pass on to the formation of the reference image, i.e. calculation of the basic matrix of the characteristic parameters of the reference image.

Let's assume that there are $l(l=1,2, \ldots)$ ethnical groups and there are $n_{l}$ processes images of male and female faces looking forward in each face bases created for these groups. Then matrix $P^{l}\left(p_{i k}^{l}, i=1,9 ; k(i, j)=1,190\right)$ of parameters of reference images for the $l^{\text {th }}$ group must be determined as following:

$$
p_{i k}^{l}=\sum_{j=1}^{n_{l}} w_{i k}^{j l} / n_{l}
$$

where $w_{i k}^{j l}$ - is the element of the matrix of characteristic parameters of the $j^{\text {th }}$ face of the $l^{\text {th }}$ group of $W^{j l}\left(w^{j l}{ }_{i k}\right.$ $i=1,9 ; k(i, j)=1,190)$.
The task of determination of affiliation of the reference image to one of the $l$ ethnical groups comes down to finding Euclidean distance among the matrix of $P^{l}\left(p_{i k}^{l}, i=1,9 ; k=1,190\right)$ of $l$ ethnical groups and the matrix of $P^{*}\left(p^{*}{ }_{i k}, \quad i=1,9 ; k(i, j)=1,190\right)$ of characteristic parameters of the reference image as following:

$$
D_{l}=\sqrt{\sum_{i=1}^{9} \sum_{k=1}^{190}\left(p_{i k}^{l}-p_{i k}^{*}\right)^{2}}, l=1,2, \ldots
$$

Further, the value of $l^{*}=\operatorname{index} D$, where $D=\min \left(D_{l}, l=1,2, ..\right)$ determines the number of the ethnical groups with which the reference image is affiliated.

\section{Conclusions}

Identification system of recognition was developed based on the proposed method and algorithm. The results of conducted experiments have shown good results for the recognition description of which is referenced in following works of the authors.

Let's note that the organization of identification system of recognition, automatic finding of identification points on the face is equivalent in the complexity of the task of recognition. In this work, the location of the identification points on portrait photograph is carried out manually by overlaying the predefined template of these points to the images.

Theoretical studies and practical results of recognition by national - racial affiliation based on the portrait photographs can be used in the interests of the many professions that require adequate recognition of people (customs, border control, security, etc.). Upon determining the identity based on identification systems, the basis of which contain several millions of portraits - will significantly narrow the search area while reducing the time of recognition.

\section{References}

[1] Adilova L.R. On face recognition//Intellectual systems, vol. 14. Moscow, 2010, pp. 73-84.

[2] Alberto Del Bimbo, Pietro Pala, Stefano Berretti, Distinguishing Facial Features for Ethnicity-Based 3D Face Recognition // ACM Transactions on Intelligent Systems and Technology (TIST), 2012, pp. 1-20.

[3] Jingu Heo, M., Savvides, Gender and Ethnicity Specific Generic Elastic Models from a Single 2D Image for Novel 2D Pose Face Synthesis and Recognition // IEEE Transactions on Pattern Analysis and Machine Intelligence (TPAMI), vol. 34, 2012, pp. 2341-2350.

[4] Guangpeng Zhang, Yunhong Wang, Multimodal 2D and 3D Facial Ethnicity Classification // Fifth International Conference on Image and Graphics. China September 20 23, 2009.

[5] Satoshi Hosoi, Erina Takikawa, Masato Kawade, Ethnicity Estimation with Facial Images / Sixth IEEE International Conference on Automatic Face and Gesture Recognition (FG'04), Seoul, Korea, May 17-19, 2004.

[6] Huaxiong Ding, Liming Chen, Facial ethnicity classification based on boosted local texture and shape descriptions / 2013 10th IEEE International Conference and Workshops on Automatic Face and Gesture Recognition (FG). Shanghai, China, April 22- 26, 2013.

[7] Kazımov T.H., Mahmudova Sh.J. About creation of system of computer recognition of people by photographs / 
Proceedings of the Fifth International Conference on Neural Networks and Artificial Intelligence, May 27-30, 2008, Minsk, pp. 164-167.

[8] Kyazimov T. G., Mahmudova Sh.Dzh. The Effectiveness Increase of a System of Automatic Biometrical Identification Based on Photo Portraits // Automatic Control and Computer Sciences, 2011,Vol. 45, No. 2, USA, pp. 106-112.

[9] Mahmudova Sh.J. Definition of weight coefficient of geometric characteristics used for identification of human face on the basis of photo-portrait / Proceedings of the 6th 2011 International Conference on Computer Sciences and Convergence Information Technology, Nov. 29 - Dec. 1, Jeju Island, Republic of Korea, IEEE Press, 2011, pp. 797802.

[10] Kazımov T.H., Mahmudova Sh.J. About a Method of
Calculation of Importance Degree of Geometrical Characteristics to Identify a Human Face on the Basis of Photo Portraits // Computer Science and Engineering, 2012,Vol. 2, No. 5, USA, pp. 59-62.

[11] Kazımov T.H., Mahmudova Sh.J. About A Method of Calculation of Importance Degree of Geometrical Characteristics to Identify a Human Face On The Basis Of Photo Portraits / Proceedings of the 7 th IAPR International Conference on Pattern Recognition in Bioinformatics, 2012, November 8-10, Tokyo, pp. 154-160.

[12] Kazımov T. H., Mahmudova Sh.J. Increase of indicator values of identification systems quality on the recognition of human face on the basis of photoportraits // Intelligent Control and Automation, 2013,Vol. 4, No. 2, USA, pp. 191 198. 